\title{
Characterization, by Size, Density, Osmotic Fragility, and Immunoaffinity, of Acetylcholine- and Vasoactive Intestinal Polypeptide-Containing Storage Particles from Myenteric Neurones of the Guinea-Pig
}

\author{
D. V. Agoston and V. P. Whittaker \\ Arbeitsgruppe Neurochemie, Max-Planck-Institut für biophysikalische Chemie, Göttingen, F.R.G.
}

\begin{abstract}
When cytoplasmic extracts of guinea-pig myenteric neurones are submitted to centrifugal density gradient fractionation in a zonal rotor acetylcholine is bimodally distributed in the gradient, in a peak (I) rich in synaptic vesicles of the classic type and in a denser peak (II/VI) rich in densecored vesicles and vasoactive intestinal polypeptide (VIP). The putative stable synaptic vesicle markers synaptophysin (p38), vesicular proteoglycan, and $\mathrm{Mg}^{2+}$-activated ATPase were also bimodally distributed, with a peak coincident with peak I and another, broader peak embracing peak II/VI, and neighbouring peaks of other neuropeptides resolved from peak II/VI by the density gradient separation procedure used. To establish whether the stable markers, acetylcholine and VIP in peak II/VI were present in one particle or several, attempts were made to separate them by particle-exclusion chromatography and differential osmotic fragility. These were unsuccessful, leading us to conclude that the storage particles
\end{abstract}

in peak II/VI contain both neurotransmitters and all three putative stable synaptic vesicle markers. It is suggested that such particles are the counterparts, in cholinergic neurones of the myenteric plexus, of the dense-cored, enkephalin- and noradrenaline-containing vesicles of certain adrenergic neurones and, like the latter, may exist in a precursor-product relationship with the classic synaptic vesicles containing the small-molecular-mass transmitters and found in the same nerve terminals. Key Words: Myenteric neurones-Characterization of storage particles rich in acetylcholine and vasoactive intestinal polypeptide-Synaptophysin-Vesiclespecific proteoglycan-MgATPase. Agoston D. V. and Whittaker V. P. Characterization, by size, density, osmotic fragility, and immunoaffinity, of acetylcholine- and vasoactive intestinal polypeptide-containing storage particles from myenteric neurones of the guinea-pig. J. Neurochem. 52, 1474-1480 (1989).
In a previous article (Agoston et al., 1985b) we observed that homogenates of the myenteric plexus-longitudinal muscle (MPLM) preparation of guinea-pig ileum prepared under conditions of relatively mild liquid shear ("protective homogenization," $\mathrm{PH}$ ) contained acetylcholine (ACh)-rich synaptic vesicles and storage particles for substance $\mathrm{P}$ (SP), somatostatin (SS), and vasoactive intestinal polypeptide (VIP), all of which could be separated from each other by high-resolution centrifugal density-gradient separation on a zonal rotor. The peaks containing the neuroactive substances were designated I (synaptic vesicles), IV (SP), V (SS), and VI (VIP). In recent work (D. V. Agoston and C. Shaw, in preparation) the presence of a fifth peak, less dense than peak IV, and containing gastrin-releasing polypeptide (GRP), has been identified. Interestingly, ACh was bimodally distributed in the gradient under PH conditions (which were also optimal for VIP-containing particles): in addition to its main peak in association with synaptic vesicles, it formed a second peak (II) which coincided with VIP. The main vesicular component in this peak (here designated II/VI) was dense cored, with a diameter of $110 \mathrm{~nm}$.

The main object of the present work was to determine whether ACh and VIP in peak II/VI really were in the same storage particle or in separate particles. For

\footnotetext{
Received August 22, 1988; accepted October 17, 1988.

Address correspondence and reprint requests to Dr. V.P. Whittaker at Arbeitsgruppe Neurochemie, Max-Planck-Institut für biophysikalische Chemie, Postfach 2841, D-3400 Göttingen, F.R.G.

The present address of Dr. D. V. Agoston is Laboratory of Cell Biology, National Institute of Mental Health, Bldg. 36 Rm. 3A17, Bethesda, MD 20892, U.S.A.
}

Abbreviations used: ACh, acetylcholine; GRP, gastrin releasing polypeptide; MgATPase, $\mathrm{Mg}^{2+}$-activated ATPase; MPLM, myenteric plexus-longitudinal muscle; $\mathrm{PG}$, putative vesicle specific proteoglycan; $\mathrm{PH}$, protective homogenization; PMSF, phenylmethylsulphonyl fluoride; SDS-PAGE, sodium dodecyl sulphate-polyacrylamide gel electrophoresis; SP, substance P; SS, somatostatin; SY, synaptophysin (p38); VIP, vasoactive intestinal polypeptide. 
this, three tests of homogeneity were applied: attempts were made to separate the two activities in modified gradients, by particle exclusion chromatography, and varying degrees of hypoosmotic stress were applied in an effort to secure differential release.

The distribution of three stable putative synaptic vesicle markers, $\mathrm{Mg}^{2+}$-activated ATPase (MgATPase; EC 3.6.1.3) (Breer et al., 1977), vesicular proteoglycan (PG) (Walker et al., 1983), and synaptophysin (p38) (SY) (Jahn et al., 1985; Wiedenmann and Franke, 1985) in fractions from density gradients or particle exclusion chromatography was measured in an attempt to detect contamination of fractions containing VIP storage particles with synaptic vesicles.

A preliminary account of this work has been given (Agoston, 1987).

\section{MATERIALS AND METHODS}

\section{Fractionation of MPLM storage particles}

Isolation of storage particles by zonal density-gradient centrifugation. The procedure of Dowe et al. (1980) and Agoston et al. $(1985 a, b)$ was followed with modifications. MPLM strips $(8-10 \mathrm{~g})$ were prepared from the ilea of six guinea-pigs of $300-350 \mathrm{~g}$ body weight and placed in $0.16 \mathrm{M} \mathrm{NaCl}$ isotonic homogenization buffer at room temperature $\left(20^{\circ} \mathrm{C}\right)$. The use of room temperature instead of ice-cold conditions prevented the cold-induced fusion of peptide-containing vesicles and so increased the yield. All solutions contained: $10 \mathrm{mM}$ HEPES, pH 6.8; $1 \mathrm{~m} M$ EGTA, $0.1 \mathrm{~m} M$ EDTA; $0.1 \mathrm{~m} M$ phenylmethylsulphonyl fluoride (PMSF); the kallikrein inhibitor aprotinin (Trasylol, Bayer), $100 \mathrm{U} \mathrm{ml}^{-1} ; 0.01 \mathrm{mM}$ $\mathrm{NaN}_{3} ; 1 \mathrm{~m} M$ dithiothreitol. In some experiments $\mathrm{NaN}_{3}$ was replaced by $0.01 \%$ thiomersal and $0.05 \%$ bacitracin was also included. The homogenization medium containing the isolated strips was replaced by $32-40 \mathrm{ml}$ of cold medium $\left(4^{\circ} \mathrm{C}\right)$ and homogenization was begun immediately. The strips were first minced with scissors for $5 \mathrm{~min}$ giving an approximately $20 \%$ (wt/vol) tissue dispersion. This was further comminuted in a high speed Virtis blender at 45,000 rpm for two $10-\mathrm{s}$ periods with a cooling interval. In some experiments Antifoam (Sigma) was added before the comminution. This prehomogenate was placed in a Teflon-glass homogenizer and 10 passes were made at $800 \mathrm{rpm}$ lasting for two 2-min periods with a cooling break. This final homogenate was centrifuged for $20 \mathrm{~min}$ at $1,000 \mathrm{gav}_{\mathrm{av}}$ at $4^{\circ} \mathrm{C}$. The supernatant was decanted and the pellet and the pellicle were rehomogenized and recentrifuged as described above. The combined supernatant was loaded onto a gradient formed in a 300-ml-capacity Beckman Ti- 60 zonal rotor as previously described by Agoston et al. $(1985 a, b)$ or onto a more linear gradient formed using $1.0 \mathrm{M}$ sucrose as the first dense solution and $1.6 \mathrm{M}$ as the second; changeover was after $200 \mathrm{ml}$. Centrifugation for both conditions was at $60,000 \mathrm{rpm}$ for $3 \mathrm{~h}$.

Particle exclusion chromatography. Peaks I and Il/VI identified by their sucrose concentrations (peak I, 0.4-0.5 M sucrose; peak II/VI, 0.95-1.2 $M$ sucrose) were pooled and chromatographed on a column of Sephacryl-1000 (Pharmacia) as described by Agoston et al. (1989). Such columns are capable of resolving synaptic vesicles from dense-cored vesicles derived from Torpedo electromotor neurones (Agoston et al., 1989) though the separation is not as effective as with density gradient centrifuging. Care was taken, by ad- justing the $\mathrm{NaCl}$ content of the $\mathrm{NaCl}-0.32 \mathrm{M}$ sucrose that comprised the mobile phase, to ensure that the osmolarities of the mobile phase during chromatography were identical to those of the pooled fractions as determined by means of a Krauss osmometer. This precaution resulted in a better $(50-65 \%)$ recovery, especially in the case of the peptide-containing vesicles. In some runs $\mathrm{NaCl}$ was replaced by the required amount of glycine; however, glycine interfered with the leech bioassay so it was used in only a limited number of experiments.

Columns $(60 \times 1 \mathrm{~cm}$ or $120 \times 1 \mathrm{~cm})$ of Sephacryl-1000 (Pharmacia) were equilibrated at a flow rate of $20 \mathrm{ml} \mathrm{h}^{-1}$ with degassed elution buffer containing: $0.32 \mathrm{M}$ sucrose; the desired amount of $\mathrm{NaCl} ; 10 \mathrm{mM}$ HEPES buffer, $\mathrm{pH} 6.6$ (at $\left.4^{\circ} \mathrm{C}\right) ; 1 \mathrm{~m} M$ EGTA; $0.1 \mathrm{~m} M$ EDTA; $0.3 \mathrm{~m} M$ PMSF; $100 \mathrm{U}$ $\mathrm{ml}^{-1}$ Trasylol (Bayer); $0.01 \%$ thiomersal; $1 \mathrm{~m} M$ dithiothreitol; and in some runs $0.05 \%$ bacitracin. After several bed volumes of wash the column was calibrated with $2-3 \mathrm{ml}$ of Torpedo electromotor synaptic vesicles prepared according to our standard procedure (Ohsawa et al., 1979). The columns were then conditioned with a $100,000 \mathrm{~g}$ supernatant of the zonal parent fraction to reduce nonspecific adsorption of subcellular particles. Pooled fractions $(4-8 \mathrm{ml})$ were applied for each run and 120 -drop (approximately $4-\mathrm{ml}$ ) fractions were collected. All preparative procedures were carried out in a cold room at $4^{\circ} \mathrm{C}$.

\section{Determination of osmotic fragility}

$\mathrm{ACh}$ - and VIP-containing fractions derived from zonal runs were pooled and samples were taken for transmitter and protein determination. The pooled fractions were then aliquoted (usually $1.0 \mathrm{ml}$ ) and exposed to dialysis against large volumes (usually $2 \mathrm{~L}$ ) of buffers with osmolarities reduced stepwise. The dialysis buffer was identical to that of the sucrose gradient except in respect to sucrose concentration. As controls, pooled fractions were dialysed against a buffer with an osmolarity identical to that of the pooled fractions. The dialysis was carried out for $6 \mathrm{~h}$ in the cold $\left(4^{\circ} \mathrm{C}\right)$. The osmolarities of the buffer present in the bags were determined at the end of the dialysis by freezing-point depression. The contents of the bags were then divided and their protein, $\mathrm{ACh}$ and VIP contents were determined. Results were expressed as the percentage of contents derived from control, isoosmotic samples. Also the volumes of each bag were measured after dialysis and dilution factors were calculated; all results have been corrected appropriately.

\section{Analytical methods}

Determination of neuroactive substances. ACh was measured by bioassay on the dorsal muscle of the leech (Whittaker and Barker, 1972). Samples were acidified to $\mathrm{pH} 4.0$ and stored at $-20^{\circ} \mathrm{C}$ until assay. VIP was extracted from the fractions by bringing them to $95^{\circ} \mathrm{C}$ by the addition of 10 volumes of boiling distilled water, maintaining them at that temperature in a water bath for $5 \mathrm{~min}$, cooling on ice and then adding acetic acid to a final concentration of $0.1 \mathrm{M}$. Samples were then lyophilized using a freeze drier equipped with a vacuum centrifuge.

VIP was assayed as described using guinea-pig VIP as a standard (Agoston et al., 1985b, 1988a,b).

Enzyme determinations. MgATPase activity was determined in the presence of oligomycin and ouabain as described by Agoston et al. (1989). $P_{i}$ was determined in protein-free supernatants according to Lanzetta et al. (1979). In some $\mathbf{P}_{\mathbf{i}}$ determinations Triton X-100 (Sigma) replaced Flaminox (Fischer), giving identical sensitivity and stability. 


\section{Immunochemical procedures}

Sample preparation and dot assay of stable vesicle markers. Particles were pelleted from zonal or chromatographic fractions in a Beckman Airfuge using multiple runs and the pellets were treated as described by Jahn et al. (1985) and Agoston et al. (1986). Samples were dotted immediately onto nitrocellulose paper (Bio-Rad, Munich, F.R.G.) or stored at $-80^{\circ} \mathrm{C}$ in aliquots until assay. To exclude disproportionality due to interference or nonspecific binding $0.5,1.0$, and $2.0 \mu \mathrm{l}$ of samples were dotted. Experimental details and calculation of results are published elsewhere (Jahn et al., 1985; Agoston et al., 1986).

Gel electrophoresis. Pellets were dissolved in sample buffer and separated electrophoretically on $10 \%$ polyacrylamide gels in the presence of sodium dodecyl sulphate (SDS-PAGE) in a mini-gel apparatus according to Laemmli (1970); the separated proteins were stained after the runs by a silver-staining procedure (for details see Agoston et al., 1985b). The stained gels were dried onto Whatman filter paper in a Bio-Rad apparatus and scanned in a Shimadzu densitometer.

Immunoprecipitation of storage particles. Immunoprecipitation was performed using the anti-synaptophysin polyclonal antibody R10 and an immunobead second antibody reagent (Bio-Rad) on pooled fractions derived from zonal separations. ACh- (peak I) and VIP- (peak II/VI) containing fractions were identified by their refractive indices and pooled. To prevent loss of transmitters during the immunoprecipitation procedure the osmolarity of the pooled fractions was maintained during the entire procedure by the addition of appropriate amounts of sucrose and $\mathrm{NaCl}$. As a result of the increased density of the incubation medium the centrifugation time was longer than recommended to ensure the sedimentation of the beads; this also resulted in a better separation of bound and free vesicles. The vesicle fractions contained: $0.1 \%$ bovine serum albumin; $1 \mathrm{mM}$ EGTA; $0.02 \%$ $\mathrm{NaN}_{3} ; 100 \mathrm{U} \mathrm{ml}^{-1}$ Trasylol (Bayer); $0.3 M$ sucrose; $10 \mathrm{~m} M$ Tris $\mathrm{HCl}, \mathrm{pH} 7.4$; and $0.16 \mathrm{M} \mathrm{NaCl}$. The suspensions were incubated overnight at $4^{\circ} \mathrm{C}$ with gentle rotation. Second antibody-coated immunobeads were washed several times before use with $0.16 \mathrm{M} \mathrm{NaCl}$ in $10 \mathrm{mM} \mathrm{Na}$-phosphate buffer, pH 7.4, containing $0.02 \% \mathrm{NaN}_{3}$. The vesicle fractions were incubated with freshly washed immunobeads for $2 \mathrm{~h}$ at room temperature. After sedimentation at $1,000 \mathrm{~g}$ for $10 \mathrm{~min}$ in the cold, supernatants were removed, and the pellets were processed for $\mathrm{ACh}$ and VIP assay. Several controls were used: (1) to test the efficacy of separation no immunobeads were added; after decanting the supernatants the tubes were processed as above for transmitter assay; (2) the first antibody was replaced by control pooled rabbit sera; (3) to test the nonspecific binding capacity of immunobeads the first antiserum was omitted.

Immunoblot analysis of separated proteins. Gels were equilibrated after electrophoresis in an electrotransfer buffer of composition: $0.19 M$ glycine; $0.025 M$ Tris, $\mathrm{pH} 8.0 ; 20 \%$ (vol/vol) methanol for $30 \mathrm{~min}$ followed by electrophoretic transfer for $60 \mathrm{~min}$ at $4^{\circ} \mathrm{C}$ (Towbin et al., 1979). Following incubations with the first antibodies ${ }^{125}$ I-labelled second antibody (anti-rabbit, affinity-purified Fab fragment, Amersham-Buchler, Braunschweig, F.R.G.) was applied. After washing and drying, the filters were exposed to Amersham Betamax $\mathrm{x}$-ray film at $-80^{\circ} \mathrm{C}$.

Antibodies. The two cholinergic-specific anti-proteoglycan antisera of Walker et al. (1983) were used as previously described by Agoston et al. (1986). The three different antisynaptophysin (p38) antibodies used in our experiments were as follows. R 10 polyclonal antibody, kindly provided by $\mathrm{Dr}$. E. Floor and characterized by Buckley et al. (1987); this was used through the experiments. In some experiments the binding of two monoclonal antibodies was also tested: SY38, a kind gift from Dr B. Wiedenmann (Wiedenmann and Franke, 1985) and C 7.2, a small amount of which was made available through Mr. J. W. Hell with the kind approval of Dr. R. Jahn (Jahn et al., 1985). All gave similar results.

Protein content of fractions. Protein content was determined by the method of Peterson (1977).

\section{RESULTS}

\section{Fractionation of storage particles}

Density-gradient centrifugation. Figure la shows the distribution of ACh and VIP in the type of gradient previously used (Agoston et al., 1986); the starting máterial was prepared by the modification of our original PH technique described in Materials and Methods. As before, particles containing VIP collect in a dense region of the gradient (peak at $1.1 \mathrm{M}$ sucrose) corresponding to peak VI of our earlier work: ACh is distributed bimodally; its main peak (I) is as before at 0.4 $M$ sucrose and a second smaller peak (II) coincides with the single VIP peak at $1.1 M$ sucrose. The shoulder at $1.2 \mathrm{M}$ sucrose observed in the VIP peak but not noted earlier was reproducible under our mild ho-

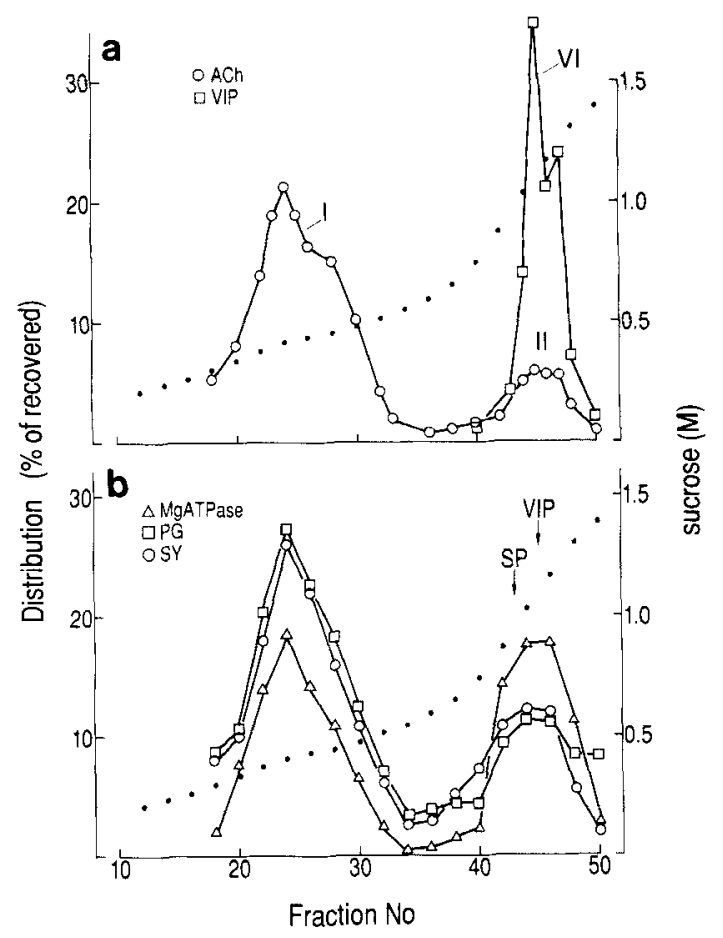

FIG. 1. Distribution (as a percentage of total recovered) of (a) $A C h$ and VIP and (b) MgATPase, PG, and SY in a sucrose density gradient after centrifugal separation in a Beckman Ti-60 zonal rotor for $3 \mathrm{~h}$ at $60,000 \mathrm{rpm}$ of a $30-\mathrm{ml}$ sample of supernatant derived from 8-10 g of MPLM. The arrows in (b) indicate the positions in the gradient of the SP and VIP peaks. The dense peak of stable markers embraces both peptide peaks. The volume of each fraction was $5 \mathrm{ml}$. 
mogenization conditions and may represent synaptosomal VIP since as we have previously shown (Dowe et al., 1980) the small number of synaptosomes generated by homogenizing MPLM sediment to this density.

Figure $1 \mathrm{~b}$ shows the distribution of MgATPase activity and PG and SY immunoreactivity. It will be seen that the distribution of these three stable vesicle markers is almost identical and is also bimodal with a peak coincident with the ACh-rich synaptic vesicle peak and a second peak overlapping peak II/VI. This suggests that these markers are common both to synaptic vesicles and the larger dense-cored vesicles in the denser part of the gradient. The broadness of the dense peak in Fig. $1 \mathrm{~b}$ may be accounted for by assuming that these markers are present in all four storage particles that have been identified in this part of the gradient, those containing (in order of increasing density) GRP, SP, SS, and VIP. The arrows in Fig. $1 \mathrm{~b}$ indicate the positions of the SP and VIP peaks; both are embraced by the enzyme activity and the immunoreactivity.

Figure 2 shows a similar experiment with a gradient profile selected to increase the resolution in the denser region of the gradient $(0.8-1.4 \mathrm{M}$ sucrose). The peaks of $\mathrm{ACh}$ (II) and VIP (VI) in the dense part of the gradient (Fig. 2a) are more spread out but remain coincident; the prominent shoulder of VIP remains and is

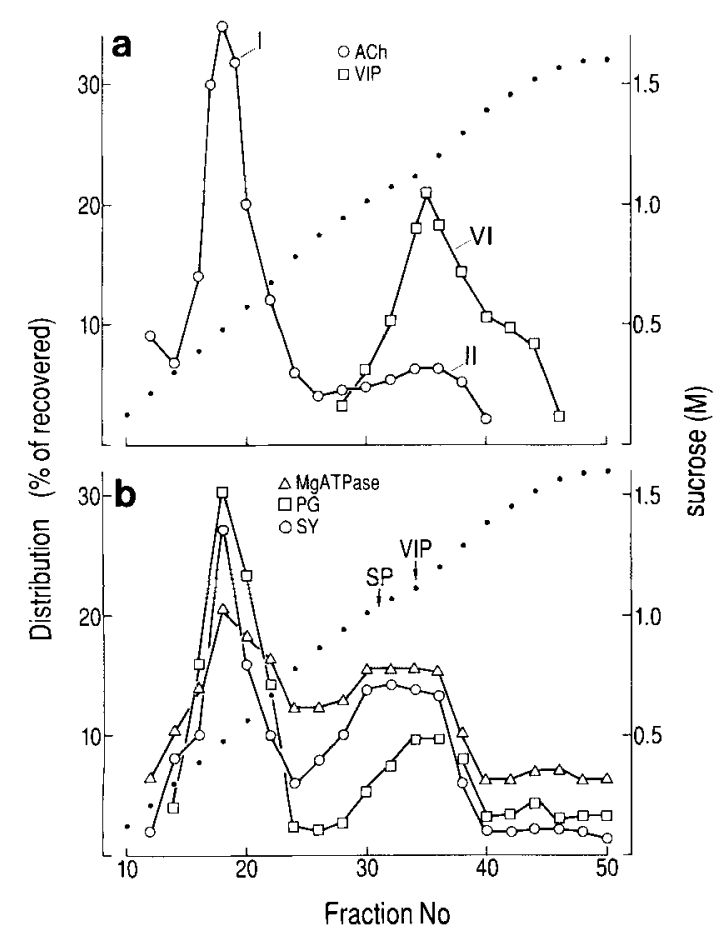

FIG. 2. An experiment similar to that shown in Fig. 1 but with a sucrose gradient that rose more steeply in its lighter region and less steeply in its denser. This had the effect of sharpening peak I and broadening peak II/VI. In spite of the increased resolution in this part of the gradient, there is no evidence for any separation of peaks II and VI. a: ACh and VIP. b: MgATPase, PG, and SY. Fraction volume as in Fig. 1.

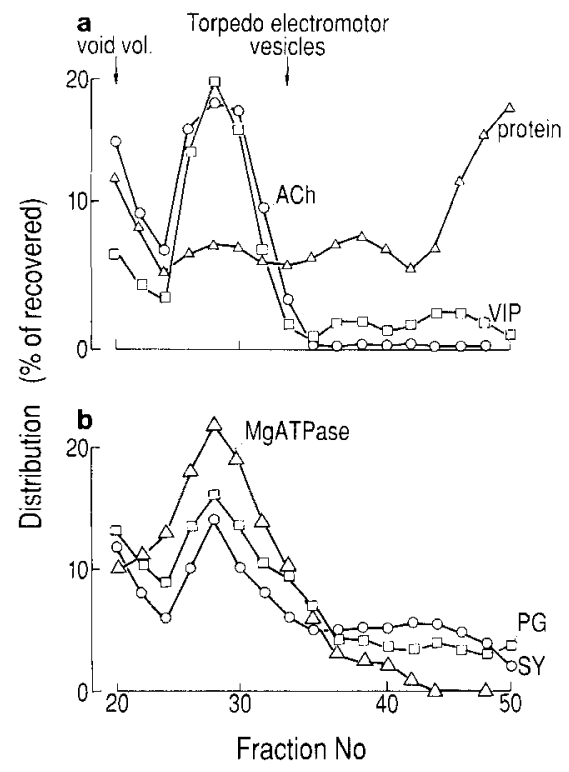

FIG. 3. Distribution of (a) ACh, VIP, and protein and (b) MgATPase, $P G$, and $S Y$ in the effluent from a $60 \times 1 \mathrm{~cm}$ column of Sephacryl1000 after loading with $4 \mathrm{ml}$ of peak II/VI. Some aggregated material came through in the void volume, but most of the ACh, VIP, MgATPase, PG, and SY emerged as a single peak with a smaller retention volume than either myenteric (peak I) or Torpedo electromotor (arrow) synaptic vesicles; thus the column failed to resolve peak II from peak VI. The final protein peak is one of soluble protein. Note that as the distribution of each component is expressed as percentage of total recovered component, and there is only one main peak, the degree of coincidence of ACh, MgATPase, PG, and SY with VIP is greater than in Figs. 1 and 2 where these four components (but not VIP) are bimodally distributed.

also broadened. Corresponding changes take place in the distribution of the stable vesicle markers. However, it is now seen that whereas the dense peaks of MgATP and $S Y$ retain their broad configuration, the distribution of PG more closely approximates to that of $\mathrm{ACh}$ and VIP.

Particle-exclusion chromatography. In these experiments possible differences in particle size rather than density were exploited in an effort to separate $\mathrm{ACh}$ from VIP. Sephacryl-1000 columns gave better results than columns of CPG-3000 B porous glass beads supplied by Electronucleotide; columns of beads provided by two other companies had insufficient resolution.

As seen in Fig. 3, chromatography of peak II/VI failed to separate $\mathrm{ACh}$ from VIP or from the stable vesicle markers; all components had an identical retention volume which was appreciably less than that of $\mathrm{ACh}$ in peak I or electromotor synaptic vesicles (arrow).

Immunoprecipitation. Because SY and PG were originally considered to be synaptic-vesicle specific, a polyclonal antibody to SY kindly provided by Dr. E. Floor and an immobilized second antibody were tested for their ability to effect the immunoprecipitation of the VIP-containing particles of peak II/VI. As a control, the same technique was applied to the ACh of peak I. 
TABLE 1. Immunoprecipitation of peaks I and VI by an anti-SY antiserum $(R \quad 10)$

\begin{tabular}{|c|c|c|c|c|}
\hline Parameter & Units & How determined & $\begin{array}{l}\text { Peak I } \\
(\mathrm{ACh})\end{array}$ & $\begin{array}{l}\text { Peak VI } \\
\text { (VIP) }\end{array}$ \\
\hline Total sedimentable activity & Percentage of total activity & $\begin{array}{l}\text { Centrifuging at } 180,000 \\
g \text { for } 1 \mathrm{~h}\end{array}$ & $87 \pm 3$ & $76 \pm 7$ \\
\hline Nonspecific immunoprecipitable activity & Percentage of sedimentable activity & Immune serum omitted & $19 \pm 3$ & $21 \pm 4$ \\
\hline Specific immunoprecipitable activity & $\begin{array}{l}\text { Percentage of sedimentable activity after deduction } \\
\text { of nonspecific immunoprecipitable activity }\end{array}$ & $\begin{array}{l}\text { Complete system } \\
\text { present }\end{array}$ & $48 \pm 6$ & $33 \pm 5$ \\
\hline
\end{tabular}

Values are means \pm SEM of four experiments.

Table 1 shows that the ACh of peak I and the VIP of peak II/VI are both precipitated in approxirnately the same proportions though the procedure was slightly less effective in immunoprecipitating VIP than ACh. This would be expected if the abundance of SY in the particles of peak VI were lower than that of peak I synaptic vesicles, or if its affinity for the antiserum were lower. However, the difference in behaviour was judged to be too small to make immunoprecipitation a valid test of the homogeneity of the VIP- and ACh-storage particles in peak II/VI.

\section{Osmotic fragility of storage particles}

Figure 4 shows the release, as a result of exposure to hypoosmotic conditions, of $\mathrm{ACh}$ from synaptic vesicles (filled circles) and from the material in peak II/ VI (open triangles), also that of VIP (filled triangles) from peak II/VI. It will be seen that the particles storing $\mathrm{ACh}$ and VIP in peak II/VI cannot be distinguished in osmotic fragility from each other, but are significantly more fragile than synaptic vesicles with their greater surface area-to-volume ratio.

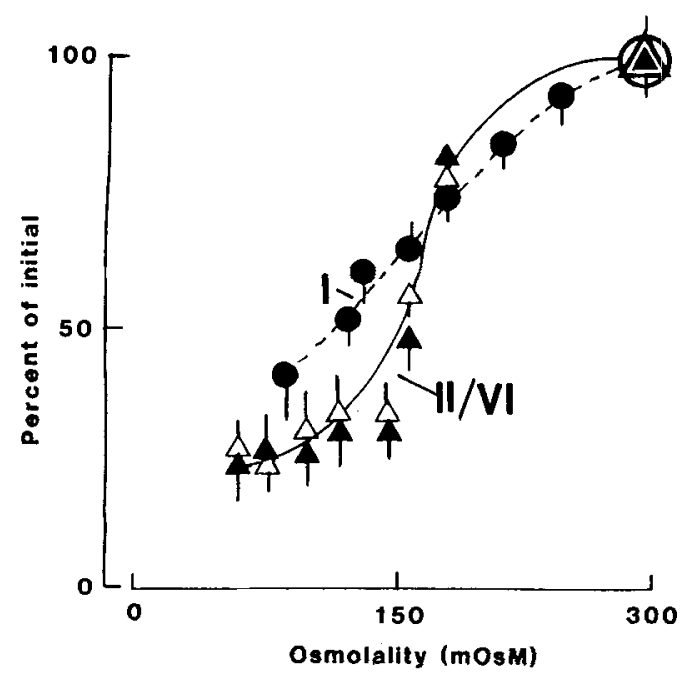

FIG. 4. Osmotic fragility of (dashed line) the synaptic vesicles of peak $I$ and of (continuous line) the particles of peak II/VI as measured by the release of ACh (filled circles and open triangles) and by VIP (filled triangles). Points and bars are means \pm SEM of four experiments.

\section{Protein composition of the two types of storage particle}

Figure 5 shows the separation, by one-dimensional SDS-PAGE, of the proteins of (left) the synaptic vesicles in peak I and (right) the particles in peak II/VI. Although the patterns are different, many common components are present, as well as the contaminants actin (A) and myosin (M) derived from the longitudinal muscle of the preparation. To the left of each gel is the result of immunostaining a "blot" of the gel with antiSY antiserum. In both gels a component of the expected mobility stains (arrows). If the staining pattern had been identical one might have argued that the presence of SY in the dense fraction was due to adsorbed, contaminating vesicles. However, the main SY component in the dense vesicle preparation has a slightly lower electrophoretic mobility, but not so low as to suggest proteolytic breakdown (Rehm et al., 1986).

\section{DISCUSSION}

The results presented here show that the particles rich in ACh and VIP that were recovered in peak II/ VI after centrifugal density gradient separation in a zonal rotor of a cytoplasmic extract of MPLM cannot be separated by further fractionation depending on putative differences in density or size; further, they have identical osmotic fragilities and are both more osmotically fragile than the ACh-rich synaptic vesicles recovered in a less dense region of the gradient. The simplest explanation of these results is that the two neuroactive substances are indeed packaged in the same, large dense-cored particle previously shown to accumulate in this region of the gradient and that the presence of $\mathrm{ACh}$ is not accounted for by contaminating synaptic vesicles.

A consequence of this interpretation of the data is that the putative synaptic vesicle markers MgATPase, $P G$, and SY can no longer be regarded as synaptic vesicle specific. The immunoblot shown in Fig. 5 shows that the SY immunoreactivity of the particles of peak II/VI differs slightly in electrophoretic migration from that of peak I, indicating that a substantial fraction of the SY of dense, neuropeptide-containing granules may be of somewhat lower molecular mass than that in synaptic vesicles, though bearing a common epitope. Our 

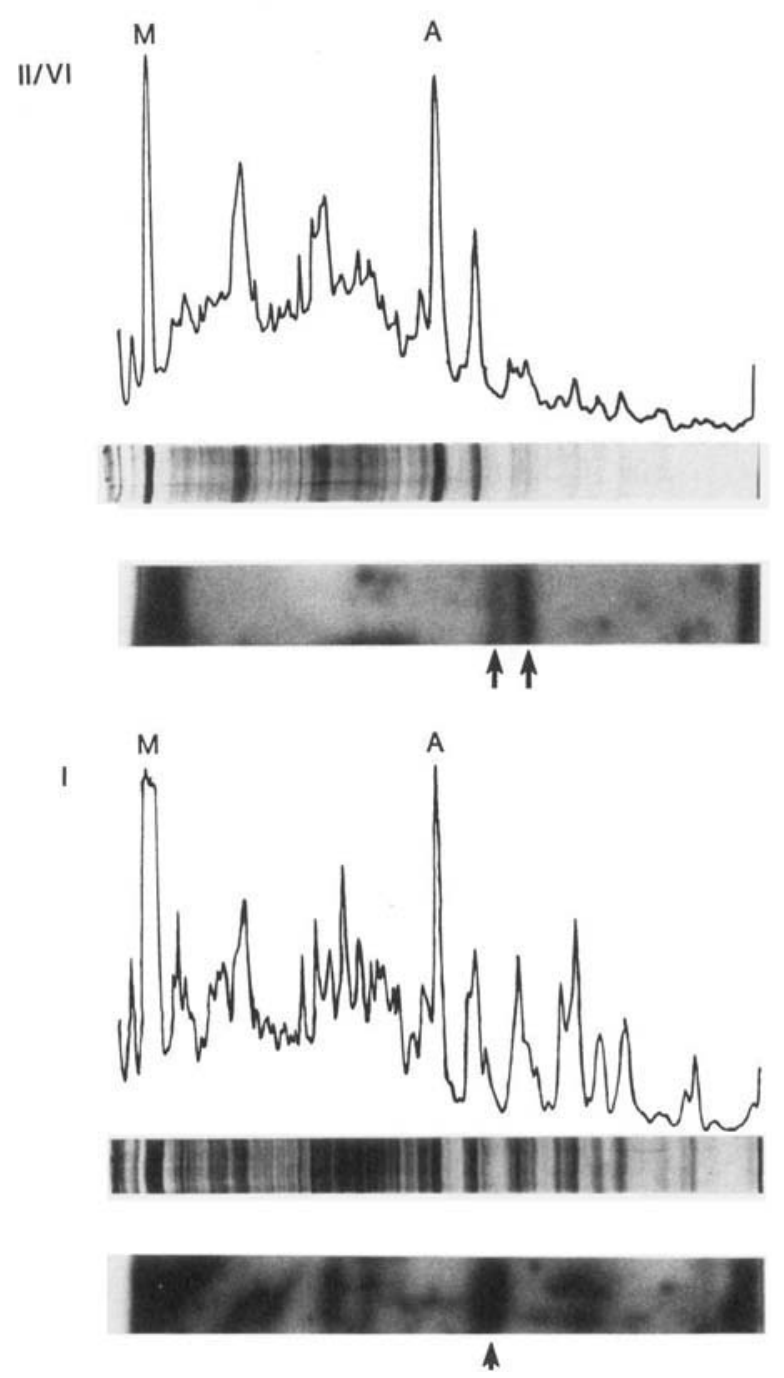

FIG. 5. One-dimensional SDS-PAGE and immunoblot of proteins of peak II/VI (upper block) and peak I (lower block) particulate material. In both blocks the central figure is the silver-stained gel; above is the densitometric scan and below the immunoblot. These indicate the presence of SY immunoreactivity in both peaks (arrows); the lower apparent molecular mass of the main immunoreactive band of peak II/VI material compared to that of peak I may indicate different extents of glycosylation of the SYs of the two particle types. $A$ and $M$ indicate actin and myosin, respectively.

experiments do not however allow us to draw conclusions about the relative abundance and affinity for antibodies of SY immunoreactivity in synaptic vesicles and dense vesicles. If SY is involved in exocytosis as some authors have suggested, it seems reasonable to expect its occurrence in any subcellular particle capable of undergoing exocytosis.

Our fractionation techniques do not allow us to conclude whether ACh-rich synaptic vesicles and dense vesicles containing both VIP and ACh coexist in the same terminals or not. However, it is tempting to believe that here we are dealing with no epiphenomenon, such as the uptake of $\mathrm{ACh}$ in response to the existence of an outwardly directed proton gradient established for other purposes (e.g., for the processing of a neuropeptide by a proton-activated cathepsin) but the physiologically significant counterpart in the cholinergic system of the occurrence, in adrenergic terminals, of two classes of noradrenaline storage particle, possibly existing in a precursor-product relationship (Geffen and Livett, 1971; Lowe et al., 1988): a large, densecored vesicle containing the neuropeptide enkephalin as well as noradrenaline, and an electron-lucent synaptic vesicle containing the low-molecular-mass transmitter alone. Here there has long been evidence for the view that the form of noradrenaline storage granule which is synthesized in the perikarya and transported down the axon is the neuropeptide-containing, densecored vesicle; at the terminal this undergoes exocytosis and its membrane, having parted company with neuropeptide and electron-dense core material alike, is retrieved as a synaptic vesicle capable of repeatedly undergoing cycles of noradrenaline uptake, exocytotic noradrenaline release, and endocytotic recovery as still functioning vesicles (for critical reviews see Lagercrantz, 1976, and Thureson-Klein, 1983). Might not the synaptic vesicles of fraction $I$, which are known to recycle (Agoston et al., 1985a), and the dense particles of fraction II/VI exist, within cholinergic neurones of the myenteric plexus, in a similar product-precursor relationship?

In a recent article (Agoston et al., 1988b) we have shown that VIP is preferentially released from MPLM strips by high-frequency stimulation and that this release is inhibited by the axonal transport blocker colchicine, whereas $\mathrm{ACh}$ is preferentially released in a colchicine-insensitive manner at low rates of stimulation. We postulated different intracellular mechanisms for the two release processes and this has been supported by other experiments (D. V. Agoston and J. Lisziewicz, submitted) in which different types of voltage-sensitive $\mathrm{Ca}^{2+}$ channels have been shown to be activated at high and low frequencies. However, these findings are not incompatible with the hypothesis that peak II/VI vesicles are the precursors of peak I vesicles, because the colchicine insensitivity of our low-frequency ACh release was tested only over a relatively short $(\sim 60 \mathrm{~min})$ period. Clearly pulse-chase labelling of a stable densecored vesicle component will be needed to settle this.

Acknowledgment: We wish to express our thanks to Mr. G. H. C. Dowe for performing the zonal runs and the acetylcholine assays, to Herrn K. Seyfert of the Klinische Arbeitsgruppe für gastrointestinale Endokrinologie der MaxPlanck-Gesellschaft (Director, Dr. J. M. Conlon) for the VIP assays, and to the Deutsche Forschungsgemeinschaft for support (Grant Wh 1/4-2).

\section{REFERENCES}

Agoston D. V. (1987) Small cholinergic and large peptidergic vesicles isolated from myenteric neurons have antigens in common. Biol. Chem. Hoppe Seyler 368, 1279.

Agoston D. V., Kosh J. W., Lisziewicz J., and Whittaker V. P. (1985a) Separation of recycling and reserve synaptic vesicles from cho- 
linergic nerve terminals of the myenteric plexus of guinea pig ileum. J. Neurochem. 44, 299-305.

Agoston D. V., Ballmann M., Conlon J. M., Dowe G. H. C., and Whittaker V. P. (1985b) Isolation of neuropeptide-containing vesicles from the guinea pig ileum. J. Neurochem. 45, 398-406.

Agoston D. V., Dowe G. H. C., Fiedler W., Giompres P. E., Roed I. S., Walker J. H., Whittaker V. P., and Yamaguchi T. (1986) A kinetic study of stimulus-induced vesicle recycling in electromotor nerve terminals using labile and stable vesicle markers. J. Neurochem. 47, 1584-1592.

Agoston D. V., Borroni E., and Richardson P. J. (1988a) Cholinergic surface antigen Chol- 1 is present in a subclass of VIP-containing rat cortical synaptosomes. J. Neurochem. 50, 1659-1662.

Agoston D. V., Conlon M. J., and Whittaker V. P. (1988b) Selective depletion of the acetylcholine and vasoactive intestinal polypeptide of the guinea-pig myenteric plexus by differential mobilization of distinct transmitter pools. Exp. Brain Res. 72, 535542.

Agoston D. V., Dowe G. H. C., and Whittaker V. P. (1989) Isolation and characterization of secretory granules storing a vasoactive intestinal polypeptide-like peptide in Torpedo cholinergic electromotor neurons. J. Neurochem. (in press).

Breer H., Morris S. J., and Whittaker V. P. (1977) Adenosine triphosphatase activity associated with purified cholinergic synaptic vesicles of Torpedo marmorata. Eur. J. Biochem. 80, 313-318.

Buckley K. M., Floor E., and Kelly R. B. (1987) Cloning and sequence analysis of cDNA encoding $\mathrm{p} 38$, a major synaptic vesicle protein. J. Cell Biol. 105, 2447-2456.

Dowe G. H. C., Kilbinger H., and Whittaker V. P. (1980) Isolation of cholinergic synaptic vesicles from the myenteric plexus of guinea-pig small intestine. J. Neurochem. 35, 993-1003.

Geffen L. and Livett B. G. (1971) Synaptic vesicles in sympathetic neurons. Physiol. Rev. 51, 98-157.

Jahn R., Schiebler W, Ouimet C., and Greengard P. (1985) A 38,000dalton membrane protein (p38) present in synaptic vesicles. Proc. Natl. Acad. Sci. USA 82, 4137-4141.

Laemmli U. K. (1970) Cleavage of structural proteins during the assembly of the head of bacteriophage T4, Nature 227, 680685 .
Lagercrantz H. (1976) On the composition and function of large dense-cored vesicles in sympathetic nerves. Neuroscience 1, 8192.

Lanzetta P. A., Alvarez L. J., Reinach P. S., and Candia O. A. (1979) An improved assay for nanomole amounts of inorganic phosphate. Anal. Biochem. 100, 95-97.

Lowe A. W., Madeddu L., and Kelly R. B. (1988) Endocrine secretory granules and neuronal synaptic vesicles have three integral membrane proteins in common. J. Cell Biol. 106, 51-59.

Ohsawa K., Dowe G. H. C., Morris S. J., and Whittaker V. P. (1979) The lipid and protein content of cholinergic synaptic vesicles from the electric organ of Torpedo marmorata purified to constant composition: implications for vesicle structure. Brain Res. $161,447-457$.

Peterson G. L. (1977) A simplification of the protein assay method of Lowry et al. which is more generally applicable. Anal. Biochem. 83, 346-356.

Rehm H., Wiedenmann B., and Betz H. (1986) Molecular characterization of synaptophysin, a major calcium-binding protein of the synaptic vesicle membrane. EMBO J. 5, 535-541.

Thureson-Klein $\AA$. (1983) Exocytosis from large and small dense cored vesicles in noradrenergic nerve terminals. Neuroscience $10,245-252$.

Towbin H., Stachelin T., and Gordon J. (1979) Electrophoretic transfer of proteins from polyacrylamide gels to nitrocellulose sheets: procedure and some applications. Proc. Natl. Acad. Sci. USA 76, 4350-4354.

Walker J. H., Obrocki J., and Zimmermann C. W. (1983) Identification of a proteoglycan antigen characteristic of cholinergic synaptic vesicles. $J$. Neurochem. 41, 209-216.

Whittaker V. P. and Barker L. A. (1972) The subcellular fractionation of brain tissue with special reference to the preparation of synaptosomes and their component organelles, in Methods of Neurochemistry, Vol. 2 (Fried R., ed), pp. 1-52. Marcel Dekker, New York.

Wiedenmann B. and Franke W. W. (1985) Identification and localization of an integral membrane glycoprotein of $\mathrm{Mr} 38000$ (synaptophysin) characteristic of presynaptic vesicles. Cell $\mathbf{4 1}$, 1017-1028. 